EPJ Web of Conferences 70, 00043 (2014)

DOI: $10.1051 /$ epjconf/ 20147000043

(C) Owned by the authors, published by EDP Sciences, 2014

\title{
The Dark Matter annual modulation results from DAMA/LIBRA
}

\author{
R. Bernabei ${ }^{1,2}$, P. Belli ${ }^{2, a}$, F. Cappella ${ }^{3,4}$, V. Caracciolo ${ }^{5}$ R. Cerulli5 ${ }^{5}$, C.J. Dai ${ }^{6}$, A. d'Angelo ${ }^{3,4}$, A. Di \\ Marco $^{1,2}$, H.L. He ${ }^{6}$, A. Incicchitti ${ }^{4}$, X.H. Ma ${ }^{6}$, F.Montecchia ${ }^{2,7}$, X.D. Sheng ${ }^{6}$, R.G. Wang ${ }^{6}$, and \\ Z.P. $Y e^{6,8}$ \\ ${ }^{1}$ Dip. di Fisica, Università di Roma "Tor Vergata", I-00133 Rome, Italy \\ 2 INFN, sez. Roma "Tor Vergata", I-00133 Rome, Italy \\ ${ }^{3}$ Dip. di Fisica, Università di Roma "La Sapienza", I-00185 Rome, Italy \\ ${ }^{4}$ INFN, sez. Roma, I-00185 Rome, Italy \\ ${ }^{5}$ Laboratori Nazionali del Gran Sasso, I.N.F.N., Assergi, Italy \\ ${ }^{6}$ IHEP, Chinese Academy, P.O. Box 918/3, Beijing 100039, China \\ ${ }^{7}$ Laboratorio Sperimentale Policentrico di Ingegneria Medica, Università degli Studi di Roma "Tor Vergata" \\ ${ }^{8}$ University of Jing Gangshan, Jiangxi, China
}

\begin{abstract}
The DAMA/LIBRA experiment, running at the Gran Sasso National Laboratory of the I.N.F.N. in Italy, has a sensitive mass of about $250 \mathrm{~kg}$ highly radiopure $\mathrm{NaI}(\mathrm{Tl})$. It is mainly devoted to the investigation of Dark Matter (DM) particles in the Galactic halo by exploiting the model independent DM annual modulation signature. The present DAMA/LIBRA experiment and the former DAMA/NaI one (the first generation experiment having an exposed mass of about $100 \mathrm{~kg}$ ) have released so far results corresponding to a total exposure of 1.17 ton $\times$ yr over 13 annual cycles. They provide a model independent evidence of the presence of DM particles in the galactic halo at 8.9 $\sigma$ C.L. on the basis of the investigated DM signature. The data of another annual cycle in the same DAMA/LIBRA running conditions are at hand. After the replacement at the end of 2012 of all the photomultipliers (PMTs) with new ones, having higher quantum efficiency, DAMA/LIBRA has entered to its phase 2; such a replacements allowed the lowering of the software energy threshold of the experiment in the present data taking. A short summary of the obtained results is presented and future perspectives of the experiment mentioned.
\end{abstract}

\section{Introduction}

The DAMA project is an observatory for rare processes located deep underground at the Gran Sasso National Laboratory of the I.N.F.N.. It is based on the development and use of low background scintillators. The main experimental set-ups are: i) DAMA/NaI ( $\simeq 100 \mathrm{~kg}$ of highly radiopure $\mathrm{NaI}(\mathrm{Tl}))$ that took data for 7 annual cycles and completed its data taking on July 2002 [1-12]; ii) DAMA/LXe, $\simeq 6.5 \mathrm{~kg}$ liquid $\mathrm{Kr}$-free Xenon enriched either in ${ }^{129} \mathrm{Xe}$ or in ${ }^{136} \mathrm{Xe}$ [13]; iii) DAMA/R\&D, a facility dedicated to tests on prototypes and to perform experiments developing and using various kinds of low background crystal scintillators to investigate various rare processes [14]; iv) DAMA/Ge, where

\footnotetext{
ae-mail: pierluigi.belli@roma2.infn.it
} 
sample measurements are carried out and where dedicated measurements on rare events are performed [15]; v) the second generation DAMA/LIBRA set-up, $\simeq 250 \mathrm{~kg}$ highly radiopure $\mathrm{NaI}(\mathrm{Tl})$ ) [16-20] mainly devoted to the investigation of the presence of Dark Matter (DM) particles in the Galactic halo. Profiting of the low background features of these set-ups, many rare processes have been studied.

Many possibilities are open as concerns the nature and the interaction types of the DM particles as e.g.: SUSY particles (as neutralino [21] or sneutrino in various scenarios [22]), inelastic Dark Matter in various scenarios [23, 24], electron interacting dark matter (including WIMP scenarios) [10], a heavy neutrino of the 4-th family, sterile neutrino [11], Kaluza-Klein particles, self-interacting dark matter, axion-like (light pseudoscalar and scalar candidate) [6], mirror dark matter in various scenarios [25], Resonant Dark Matter [26], DM from exotic 4th generation quarks [27], Elementary Black holes, Planckian objects, Daemons, Composite DM [28], Light scalar WIMP through Higgs portal [29], Complex Scalar Dark Matter [30], specific two Higgs doublet models, exothermic DM [31], Secluded WIMPs [32], Asymmetric DM [33], Isospin-Violating Dark Matter [34], Singlet DM [35], Specific GU [36], SuperWIMPs [37], WIMPzilla [38], etc. [39]. Moreover, even a suitable particle not yet foreseen by theories could be the solution or one of the solutions. Often WIMP is adopted as a synonymous of Dark Matter particle, referring usually to a particle with spin-independent elastic scattering on nuclei. On the contrary, WIMP identifies a class of Dark Matter candidates which can have different phenomenologies and interaction types. Different phenomenologies and interactions are also possible when considering a precise candidate as for example the neutralino; in fact, the basic supersymmetric theory has a large number of unknown parameters and, depending on the assumptions, they can present well different features. It is worth noting that often constrained SUGRA models (which allow easier calculations for the predictions e.g. at accelerators) are presented as SUSY or as the only way to SUSY, which is not the case. Other open aspects, which have large impact on model dependent investigations and comparisons, are e.g. which is the right description of the dark halo and related parameters, which is the right related atomic/nuclear and particle physics, etc. as well as the fundamental question on how many kinds of Dark Matter particles exist in the Universe ${ }^{1}$.

Depending on the DM candidate, the interaction processes can be various, as e.g: 1) elastic scatterings on target nuclei with either spin-independent or spin-dependent or mixed coupling; moreover, additional electromagnetic contribution in case of few $\mathrm{GeV}$ candidates can arise from excitation of bound electrons by the recoiling nuclei [8]; 2) inelastic scatterings on target nuclei with either spinindependent or spin-dependent or mixed coupling in various scenarios [23, 40]; 3) interaction of light DM (LDM) either on electrons or on nuclei with production of a lighter particle [11]; 4) preferred interaction with electrons [10]; 5) conversion of DM particles into electromagnetic radiation [6]; 6) etc.

As concerns the experiments at accelerators, they could prove the existence of some possible Dark Matter candidate particles - when a solid model independent result would be stated - while they could never credit by themselves that a certain particle is in the halo as the solution or the only solution for Dark Matter particle(s). Moreover, Dark Matter candidate particles and scenarios (even for neutralino candidate) exist which cannot be investigated at accelerators.

Therefore, considering the richness of particle possibilities and the existing uncertainties on related astrophysical (e.g. halo model and related parameters, etc.), nuclear (e.g. form factors, spin factors, scaling laws, etc.) and particle physics (e.g. particle nature and interaction types, etc.), a

\footnotetext{
${ }^{1}$ Consider the richness in particles of the luminous Universe which is just 0.007 of the density of the Universe with respect to about 0.22 of the Dark Matter attributed to relic particles by the combination of the results of WMAP, of the SN type IA and clusters observations.
} 
widely-sensitive model independent approach with a suitable exposure, and full control of the running condition over the whole data taking, is needed.

Indeed, most of the activities has released marginal exposures even after running many years underground and has based the analysis on a particular "a-priori" assumption on the nature of the DM particle and of its interaction. The halo is assumed to be isothermal, which is for many reasons unlike; moreover, all the theoretical and experimental parameters are set to some assumed fixed values.

Originally the so-called "traditional" approach was pursued by simply comparing the measured counting rate with an expectation from an assumed scenario (which implies to adopt many assumptions and approximations). This is the only approach which can be pursued by small scale or poor duty cycle experiments. More recently, large data selections and several subtraction procedures are often applied to derive a set of recoil-like candidates assuming a priori the interaction type of the DM candidate. It is worth noting that not only many uncertainties in the applied procedures and related efficiencies can exist, but well known side reactions exist able to give similar recoil-like candidates surviving the applied subtractions. In particular, e.g. the applied subtraction procedures are statistical and the tails of the subtracted populations can give rise to events indistinguishable from recoils as well (recoil-like candidates). Considering that the stability of the running conditions and of the many applied "subtraction windows" is never suitably proved at the needed level of precision, the surviving candidates can never be stated for sure as recoils (induced by neutrons, Dark Matter or whatever else) and any estimation of surviving background events cannot be achieved at the claimed levels of precision.

Although often the limits achieved by this approach have been presented as robust reference points, it can be easily understood that similar results are quite uncertain not only because of possible underestimated or unknown systematics in the large data selections and in some experimental aspects, but also because the results refer only to a certain (generally largely arbitrary) set of assumptions. The accounting of the many existing experimental and theoretical uncertainties can significantly vary the given model dependent results.

In conclusion, as mentioned suitable experiments offering a model independent signature for the presence of Dark Matter particles in the galactic halo are mandatory. At present the only feasible one is the DM annual modulation signature, originally suggested in [41] and exploited by the DAMA experiments at the Gran Sasso National Laboratory by using highly radiopure NaI(Tl) set-ups as target-detectors; in the following the already-achieved results are shortly summarized.

\section{The DAMA/LIBRA experiment}

The second generation DAMA/LIBRA set-up, $\simeq 250 \mathrm{~kg}$ highly radiopure $\mathrm{NaI}(\mathrm{Tl})$ ) [16-20], is investigating the presence of DM particles in the galactic halo by exploiting the model independent DM annual modulation signature.

In fact, as a consequence of its annual revolution around the Sun, which is moving in the Galaxy traveling with respect to the Local Standard of Rest towards the star Vega near the constellation of Hercules, the Earth should be crossed by a larger flux of Dark Matter particles around $~ 2$ June (when the Earth orbital velocity is summed to the one of the solar system with respect to the Galaxy) and by a smaller one around $\sim 2$ December (when the two velocities are subtracted). Thus, this signature has a different origin and peculiarities than the seasons on the Earth and than effects correlated with seasons (consider the expected value of the phase as well as the other requirements listed below). This DM annual modulation signature is very distinctive since the effect induced by DM particles must simultaneously satisfy all the following requirements: (1) the rate must contain a component modulated according to a cosine function; (2) with one year period; (3) with a phase that peaks 
roughly around $\sim 2$ nd June; (4) this modulation must be present only in a well-defined low energy range, where DM particles can induce signals; (5) it must be present only in those events where just a single detector, among all the available ones in the used set-up, actually "fires" (single-hit events), since the probability that DM particles experience multiple interactions is negligible; (6) the modulation amplitude in the region of maximal sensitivity has to be $\lesssim 7 \%$ in case of usually adopted halo distributions, but it may be significantly larger in case of some particular scenarios such as e.g. those in refs. [40, 42].

Only systematic effects or side reactions able to simultaneously fulfill all the six requirements given above and to account for the whole observed modulation amplitude might mimic this DM signature; no one has been found or suggested by anyone over more than a decade. Thus, no other effect investigated so far in the field of rare processes offers a so stringent and unambiguous signature.

This offers an efficient model independent signature, able to test a large number of DM candidates, a large interval of cross sections and of halo densities. At present status of technology it is the only model independent signature available in direct Dark Matter investigation that can be effectively exploited.

It is worth noting that the corollary questions related to the exact nature of the DM particle(s) (detected by means of the DM annual modulation signature) and to the astrophysical, nuclear and particle Physics scenarios require subsequent model dependent corollary analyses, as those performed e.g. in refs. [4-11]. On the other hand, one should stress that it does not exist any approach in direct and indirect DM searches which can offer information on the nature of the candidate in a model independent way, that is without assuming any astrophysical, nuclear and particle Physics scenarios.

\section{DAMA/LIBRA results}

The DAMA/NaI set up and its performances are described in ref.[1, 3-5], while the DAMA/LIBRA set-up and its performances are described in ref. [16]. The sensitive part of the DAMA/LIBRA set-up is made of 25 highly radiopure $\mathrm{NaI}(\mathrm{Tl})$ crystal scintillators placed in a 5-rows by 5-columns matrix; each crystal is coupled to two low background photomultipliers working in coincidence at single photoelectron level. The detectors are placed inside a sealed copper box continuously flushed with HP nitrogen and surrounded by a low background and massive shield made of $\mathrm{Cu} / \mathrm{Pb} / \mathrm{Cd}$ foils/polyethylene/paraffin; moreover, about $1 \mathrm{~m}$ concrete (made from the Gran Sasso rock material) almost fully surrounds (mostly outside the barrack) this passive shield, acting as a further neutron moderator. The installation has a 3-levels sealing system which excludes the detectors from environmental air. The whole installation is air-conditioned and the temperature is continuously monitored and recorded. The detectors' responses range from 5.5 to 7.5 photoelectrons $/ \mathrm{keV}$. Energy calibrations with $\mathrm{X}$-rays $/ \gamma$ sources are regularly carried out down to few $\mathrm{keV}$ in the same conditions as the production runs. In the data analysis a software energy threshold of $2 \mathrm{keV}$ is considered.

The DAMA/LIBRA data released so far correspond to six annual cycles for an exposure of 0.87 ton $\times \mathrm{yr}[17,18]$. Considering these data together with those previously collected by DAMA/NaI over 7 annual cycles $(0.29$ ton $\times y r)$, the total exposure collected over 13 annual cycles is 1.17 ton $\times y$; this is orders of magnitude larger than the exposures typically collected in the field. Several analyses on the model-independent DM annual modulation signature have been performed (see Refs. [17, 18] and references therein); here just few arguments are mentioned. In particular, Fig. 1 shows the time behaviour of the experimental residual rates of the single-hit events collected by DAMA/NaI and by DAMA/LIBRA in the (2-6) keV energy interval [17, 18]. The superimposed curve is the cosinusoidal function: $A \cos \omega\left(t-t_{0}\right)$ with a period $T=\frac{2 \pi}{\omega}=1 \mathrm{yr}$, with a phase $t_{0}=152.5$ day (June $2^{\text {nd }}$ ), and modulation amplitude, $A$, obtained by best fit over the 13 annual cycles. The hypothesis of absence 


\section{2-6 keV}

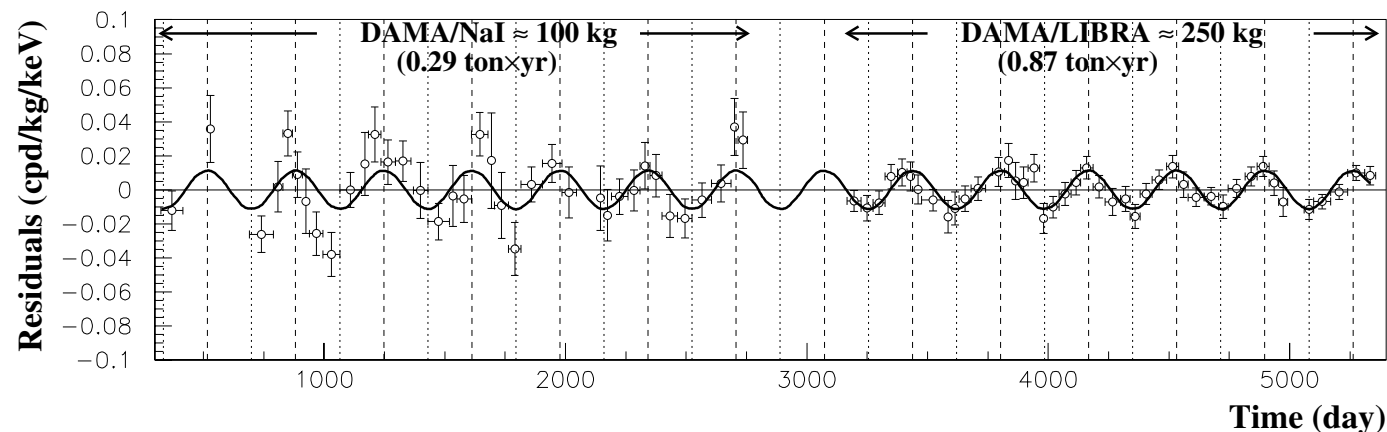

Figure 1. Experimental model-independent residual rate of the single-hit scintillation events, measured by DAMA/NaI over seven and by DAMA/LIBRA over six annual cycles in the $(2-6) \mathrm{keV}$ energy interval as a function of the time $[4,5,17,18]$. The zero of the time scale is January $1^{\text {st }}$ of the first year of data taking. The experimental points present the errors as vertical bars and the associated time bin width as horizontal bars. The superimposed curve is $A \cos \omega\left(t-t_{0}\right)$ with period $T=\frac{2 \pi}{\omega}=1 \mathrm{yr}$, phase $t_{0}=152.5$ day (June $2^{\text {nd }}$ ) and modulation amplitude, $A$, equal to the central value obtained by best fit over the whole data: cumulative exposure is 1.17 ton $\times$ yr. The dashed vertical lines correspond to the maximum expected for the DM signal (June $2^{\text {nd }}$ ), while the dotted vertical lines correspond to the minimum. See Refs. $[17,18]$ and text.

of modulation in the data can be discarded $[17,18]$ and, when the period and the phase are released in the fit, values well compatible with those expected for a DM particle induced effect are obtained [18]; for example, in the cumulative (2-6) keV energy interval: $A=(0.0116 \pm 0.0013) \mathrm{cpd} / \mathrm{kg} / \mathrm{keV}$, $T=(0.999 \pm 0.002) \mathrm{yr}$ and $t_{0}=(146 \pm 7)$ day. Summarizing, the analysis of the single-hit residual rate favours the presence of a modulated cosine-like behaviour with proper features at $8.9 \sigma$ C.L.[18].

The same data of Fig.1 have also been investigated by a Fourier analysis, obtaining a clear peak corresponding to a period of 1 year [18]; this analysis in other energy regions shows instead only aliasing peaks. Moreover, while in the (2-6) $\mathrm{keV}$ single-hit residuals a clear modulation is present, it is absent at energies just above [18]. In particular, in order to verify absence of annual modulation in other energy regions and, thus, to also verify the absence of any significant background modulation, the energy distribution measured during the data taking periods in energy regions not of interest for DM detection has also been investigated. In fact, the background in the lowest energy region is essentially due to "Compton" electrons, X-rays and/or Auger electrons, muon induced events, etc., which are strictly correlated with the events in the higher energy part of the spectrum; thus, if a modulation detected in the lowest energy region would be due to a modulation of the background (rather than to a signal), an equal or larger modulation in the higher energy regions should be present. The data analyses have allowed to exclude the presence of a background modulation in the whole energy spectrum at a level much lower than the effect found in the lowest energy region for the single-hit events [18]. A further relevant investigation has been done by applying the same hardware and software procedures, used to acquire and to analyse the single-hit residual rate, to the multiple-hits events in which more than one detector "fires". In fact, since the probability that a DM particle interacts in more than one detector is negligible, a DM signal can be present just in the single-hit residual rate. Thus, this allows the study of the background behaviour in the same energy interval of the observed positive effect. The result of the analysis is reported in Fig. 2 where it is shown the residual rate of the single-hit events measured over the six DAMA/LIBRA annual cycles, as collected in a single annual cycle, together 


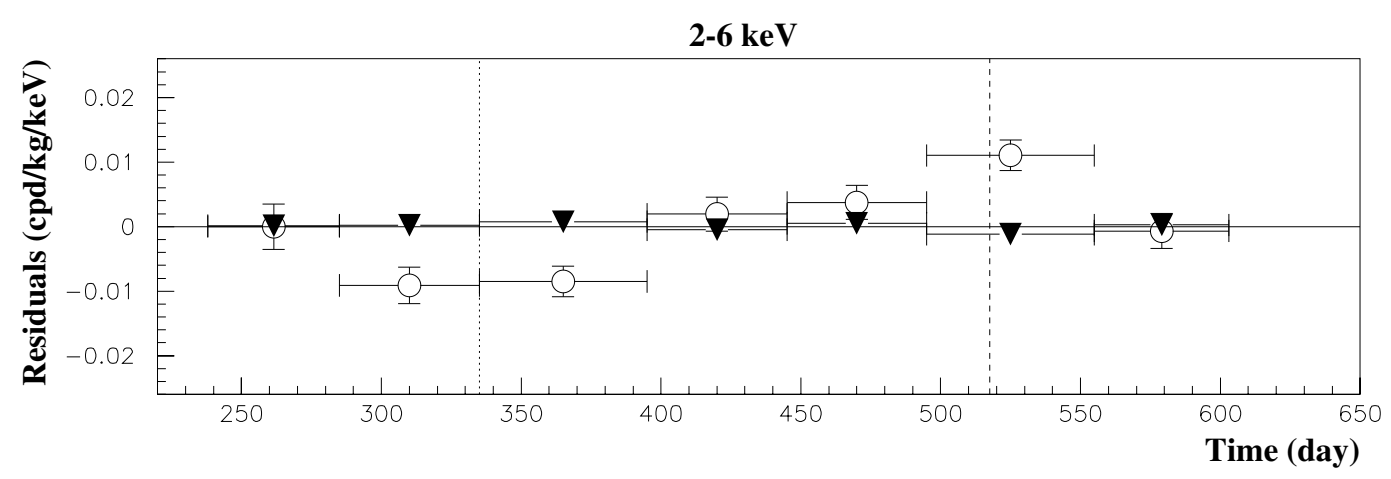

Figure 2. Experimental residual rates over the six DAMA/LIBRA annual cycles for single-hit events (open circles) (class of events to which DM events belong) and for multiple-hit events (filled triangles) (class of events to which DM events do not belong). They have been obtained by considering for each class of events the data as collected in a single annual cycle and by using in both cases the same identical hardware and the same identical software procedures. The initial time of the figure is taken on August $7^{\text {th }}$. The experimental points present the errors as vertical bars and the associated time bin width as horizontal bars. See text and Refs. [17, 18].

with the residual rates of the multiple-hits events, in the same considered energy interval. A clear modulation is present in the single-hit events, while the fitted modulation amplitudes for the multiplehits residual rate are well compatible with zero [18]. Similar results were previously obtained also for the DAMA/NaI case [5]. Thus, again evidence of annual modulation with proper features, as required by the DM annual modulation signature, is present in the single-hit residuals (events class to which the DM particle induced events belong), while it is absent in the multiple-hits residual rate (event class to which only background events belong). Since the same identical hardware and the same identical software procedures have been used to analyse the two classes of events, the obtained result offers an additional strong support for the presence of a DM particle component in the galactic halo further excluding any side effect either from hardware or from software procedures or from background.

The annual modulation present at low energy has also been analyzed by depicting the differential modulation amplitudes, $S_{m}$, as a function of the energy; the $S_{m}$ is the modulation amplitude of the modulated part of the signal obtained by maximum likelihood method over the data, considering $T=1 \mathrm{yr}$ and $t_{0}=152.5$ day. The $S_{m}$ values are reported as function of the energy in Fig. 3 . It can be inferred that a positive signal is present in the (2-6) keV energy interval, while $S_{m}$ values compatible with zero are present just above; in particular, the $S_{m}$ values in the (6-20) keV energy interval have random fluctuations around zero with $\chi^{2}$ equal to 27.5 for 28 degrees of freedom. It has been also verified that the measured modulation amplitudes are statistically well distributed in all the crystals, in all the annual cycles and energy bins; these and other discussions can be found in ref. [18].

It is also interesting the results of the analysis performed by releasing the assumption of a phase $t_{0}=152.5$ day in the procedure of maximum likelihood to evaluate the modulation amplitudes from the data of the seven annual cycles of DAMA/NaI and the six annual cycles of DAMA/LIBRA. In this case alternatively the signal has been written as: $S_{0, k}+S_{m, k} \cos \omega\left(t-t_{0}\right)+Z_{m, k} \sin \omega\left(t-t_{0}\right)=$ $S_{0, k}+Y_{m, k} \cos \omega\left(t-t^{*}\right)$, where $S_{0, k}$ and $S_{m, k}$ are the constant part and the modulation amplitude of the signal in $k$-th energy interval. Obviously, for signals induced by DM particles one would expect: i) $Z_{m, k} \sim 0$ (because of the orthogonality between the cosine and the sine functions); ii) 


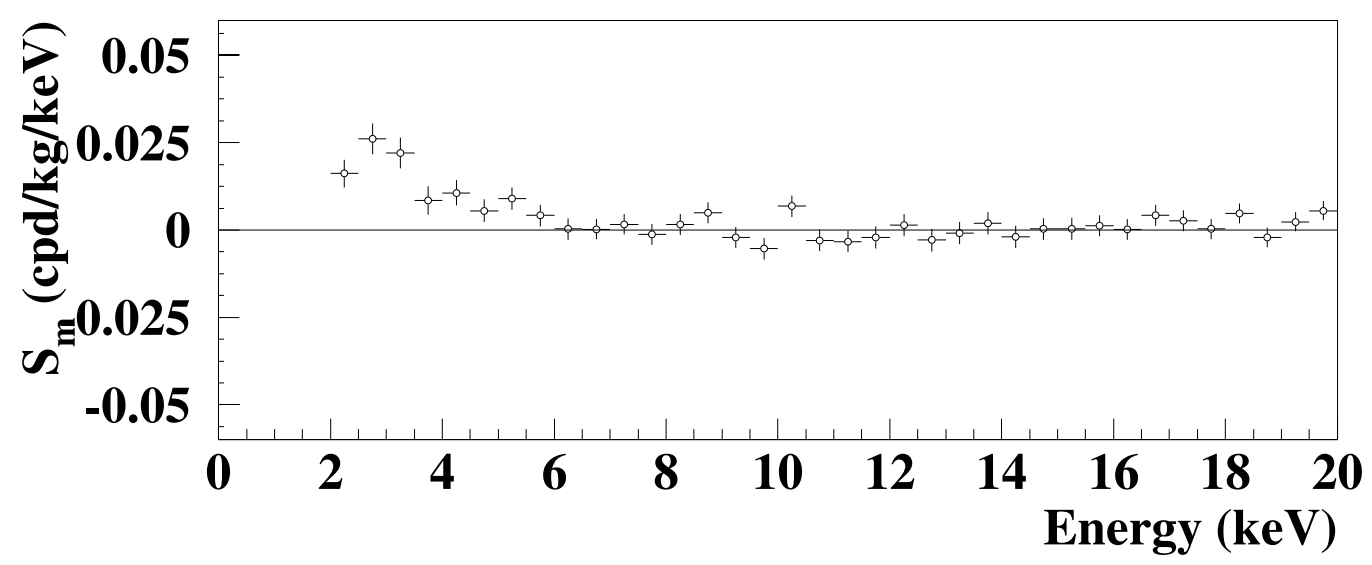

Figure 3. Energy distribution of the modulation amplitudes $S_{m}$ for the total cumulative exposure 1.17 ton $\times$ yr. The energy bin is $0.5 \mathrm{keV}$. A clear modulation is present in the lowest energy region, while $S_{m}$ values compatible with zero are present just above. In fact, the $S_{m}$ values in the (6-20) keV energy interval have random fluctuations around zero with $\chi^{2}$ equal to 27.5 for 28 degrees of freedom. See Refs. [17, 18].

$S_{m, k} \simeq Y_{m, k}$; iii) $t^{*} \simeq t_{0}=152.5$ day. In fact, these conditions hold for most of the dark halo models; however, it is worth noting that slight differences in the phase could be expected in case of possible contributions from non-thermalized DM components, such as e.g. the SagDEG stream [7] and the caustics [43]. The $2 \sigma$ contours in the plane $\left(S_{m}, Z_{m}\right)$ for the (2-6) keV and (6-14) keV energy intervals and those in the plane $\left(Y_{m}, t^{*}\right)$ are reported in Fig. 4 [18]. The best fit values for the (2-6) $\mathrm{keV}$ energy interval are $(1 \sigma$ errors $): S_{m}=(0.0111 \pm 0.0013) \mathrm{cpd} / \mathrm{kg} / \mathrm{keV} ; Z_{m}=-(0.0004 \pm 0.0014)$ $\mathrm{cpd} / \mathrm{kg} / \mathrm{keV} ; Y_{m}=(0.0111 \pm 0.0013) \mathrm{cpd} / \mathrm{kg} / \mathrm{keV} ; t^{*}=(150.5 \pm 7.0)$ day; while for the $(6-14) \mathrm{keV}$ energy interval are: $S_{m}=-(0.0001 \pm 0.0008) \mathrm{cpd} / \mathrm{kg} / \mathrm{keV} ; Z_{m}=(0.0002 \pm 0.0005) \mathrm{cpd} / \mathrm{kg} / \mathrm{keV}$; $Y_{m}=-(0.0001 \pm 0.0008) \mathrm{cpd} / \mathrm{kg} / \mathrm{keV}$ and $t^{*}$ obviously not determined. These results confirm those achieved by other kinds of analyses. In particular, a modulation amplitude is present in the lower energy intervals and the period and the phase agree with those expected for DM induced signals. For more detailed discussions see ref. [18]

Both the data of DAMA/LIBRA and of DAMA/NaI fulfil all the requirements of the DM annual modulation signature.

Sometimes naive statements were put forwards as the fact that in nature several phenomena may show some kind of periodicity. It is worth noting that the point is whether they might mimic the annual modulation signature in DAMA/LIBRA (and former DAMA/NaI), i.e. whether they might be not only quantitatively able to account for the observed modulation amplitude but also able to contemporaneously satisfy all the requirements of the DM annual modulation signature; the same is also for side reactions.

Careful investigations on absence of any significant systematics or side reaction able to account for the measured modulation amplitude and to simultaneously satisfy all the requirements of the signature have been quantitatively carried out (see e.g. ref. [4, 5, 17, 44-47], refs therein). No systematics or side reactions able to mimic the signature (that is, able to account for the measured modulation amplitude and simultaneously satisfy all the requirements of the signature) has been found or suggested by anyone over more than a decade. In Table 1 cautious upper limits (90\% C.L.) on the possible contributions to the DAMA/LIBRA measured modulation amplitude have been estimated [17]. 

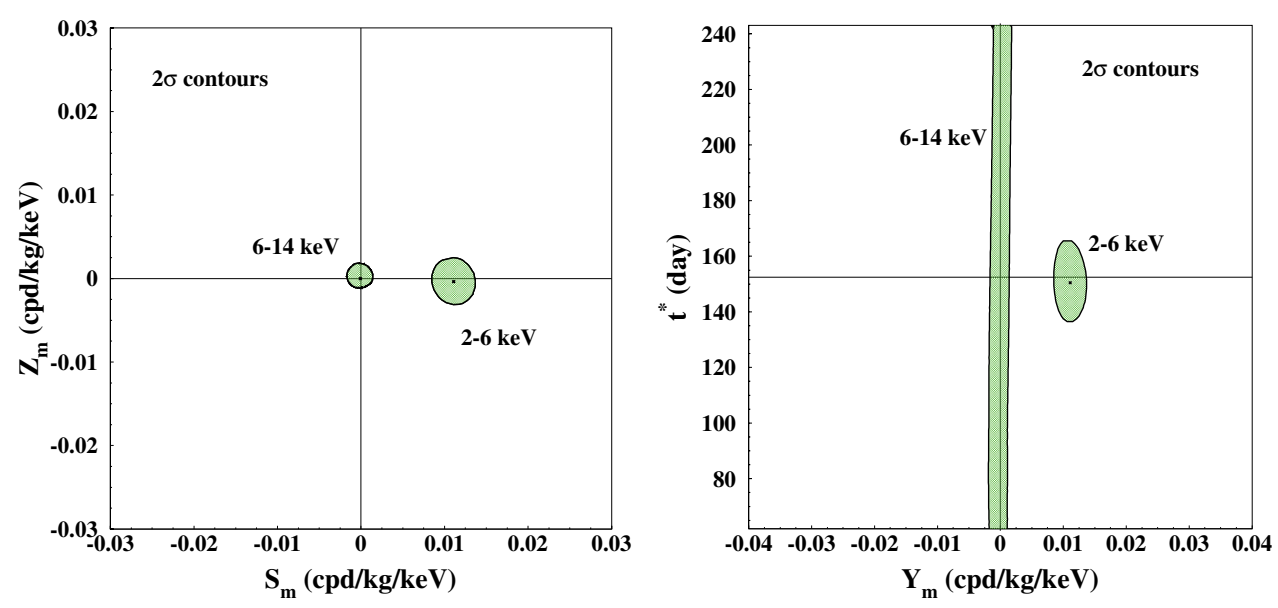

Figure 4. $2 \sigma$ contours in the plane $\left(S_{m}, Z_{m}\right)$ (left) and in the plane $\left(Y_{m}, t^{*}\right)(r i g h t)$ for the (2-6) keV and (6-14) $\mathrm{keV}$ energy intervals. The contours have been obtained by the maximum likelihood method, considering the cumulative exposure of 1.17 ton $\times$ yr. A modulation amplitude is present in the lower energy intervals and the phase agrees with that expected for DM induced signals.

The obtained model independent evidence is compatible with a wide set of scenarios regarding the nature of the DM candidate and related astrophysical, nuclear and particle Physics. For examples some given scenarios and parameters are discussed e.g. in Refs. [2, 4-11] and in Appendix A of Ref. [17]. Further large literature is available on the topics [49]; other possibilities are open. Here we just recall the recent papers $[48,50]$ where the DAMA/NaI and DAMA/LIBRA results, which fulfill all the many peculiarities of the model independent DM annual modulation signature, are examined under the particular hypothesis of a light-mass DM candidate particle interacting with the detector nuclei by coherent elastic process. In particular, in Ref. [48] allowed regions are given for DM candidates interacting by elastic scattering on nuclei including some of the existing uncertainties; comparison with theoretical expectations for neutralino candidate and with the recent possible positive hint by CoGeNT [51] is also discussed there (see Fig. 5), while comparison with possible positive hint by Cresst [52] is discussed in Ref. [50].

It is worth noting that no experiment exists, whose result can be directly compared in a modelindependent way with those by DAMA/NaI and DAMA/LIBRA.

Some activities (e.g. [53-55]) claim model-dependent exclusion under many largely arbitrary assumptions (see for example discussions in $[4,5,17,56,57]$ ); often some critical points also exist in their experimental aspects (e.g. use of marginal exposures, determination of the energy threshold, of the energy resolution and of the energy scale in the few keV energy region of interest, multiple selection procedures, non-uniformity of the detectors response, absence of suitable periodical calibrations in the same running conditions and in the claimed low energy region, stabilities, tails/overlapping of the populations of the subtracted events and of the considered recoil-like ones, well known side processes mimicking recoil-like events, etc.); moreover, the existing experimental and theoretical uncertainties are generally not considered in their presented model dependent result. Moreover, implications of the DAMA results are generally presented in incorrect/partial/unupdated way. 
Table 1. Summary of the results obtained by investigating possible sources of systematics or side processes [16-18, 44-46]. None able to give a modulation amplitude different from zero has been found; thus cautious upper limits (90\% C.L.) on the possible contributions to the measured modulation amplitude have been calculated and are shown here for the six annual cycles of DAMA/LIBRA as done before for the seven annual cycles of DAMA/NaI $[4,5]$.

\begin{tabular}{|c|c|c|}
\hline Source & Main comment & $\begin{array}{l}\text { Cautious upper limit } \\
\text { (90\% C.L.) }\end{array}$ \\
\hline Radon & $\begin{array}{c}\text { Sealed } \mathrm{Cu} \text { Box in } \\
\text { HP Nitrogen atmosphere, } \\
\text { 3-level of sealing }\end{array}$ & $<2.5 \times 10^{-6} \mathrm{cpd} / \mathrm{kg} / \mathrm{keV}$ \\
\hline Temperature & $\begin{array}{c}\text { Air conditioning } \\
+ \text { huge heat capacity }\end{array}$ & $<10^{-4} \mathrm{cpd} / \mathrm{kg} / \mathrm{keV}$ \\
\hline Noise & Efficient rejection & $<10^{-4} \mathrm{cpd} / \mathrm{kg} / \mathrm{keV}$ \\
\hline Energy scale & $\begin{array}{c}\text { Routine } \\
+ \text { intrinsic calibrations }\end{array}$ & $<1-2 \times 10^{-4} \mathrm{cpd} / \mathrm{kg} / \mathrm{keV}$ \\
\hline Efficiencies & Regularly measured & $<10^{-4} \mathrm{cpd} / \mathrm{kg} / \mathrm{keV}$ \\
\hline Background & $\begin{array}{l}\text { No modulation above } 6 \mathrm{keV} \\
\text { no modulation in the }(2-6) \mathrm{keV} \\
\text { multiple-hit events; } \\
\text { this limit includes all possible } \\
\text { sources of background }\end{array}$ & $<10^{-4} \mathrm{cpd} / \mathrm{kg} / \mathrm{keV}$ \\
\hline Side reactions & $\begin{array}{c}\text { From muon flux variation } \\
\text { measured by MACRO }\end{array}$ & $<3 \times 10^{-5} \mathrm{cpd} / \mathrm{kg} / \mathrm{keV}$ \\
\hline
\end{tabular}

As regards the model dependent indirect searches their results are restricted to some DM candidates and physical scenarios under particular specific assumptions, and require also the modeling of the existing and largely unknown competing background for the secondary particles they are looking for. No quantitative comparison can be directly performed between the results obtained in direct and indirect searches because it strongly depends on assumptions and on the considered model framework. In particular, a comparison would always require the calculation and the consideration of all the possible configurations for each given particle model (e.g., for neutralino: in the allowed parameters space), as a biunivocal correspondence between the observables in the two kinds of experiments does not exist. Therefore, neither negative nor possible positive indications are in conflict with the DAMA model independent result.

\section{Upgrades and perspectives}

A first upgrade of the DAMA/LIBRA set-up was performed in September 2008. One detector was recovered by replacing a broken PMT and a new optimization of some PMTs and HVs was done. The transient digitizers were replaced with new ones, having better performances and a new DAQ with optical read-out was installed.

A further and more important upgrade has been performed in the end of 2010 when all the PMTs have been replaced with new ones having higher quantum efficiency; details on the reached performances are reported in Ref. [58]. The purpose of the last upgrade of the running second generation 


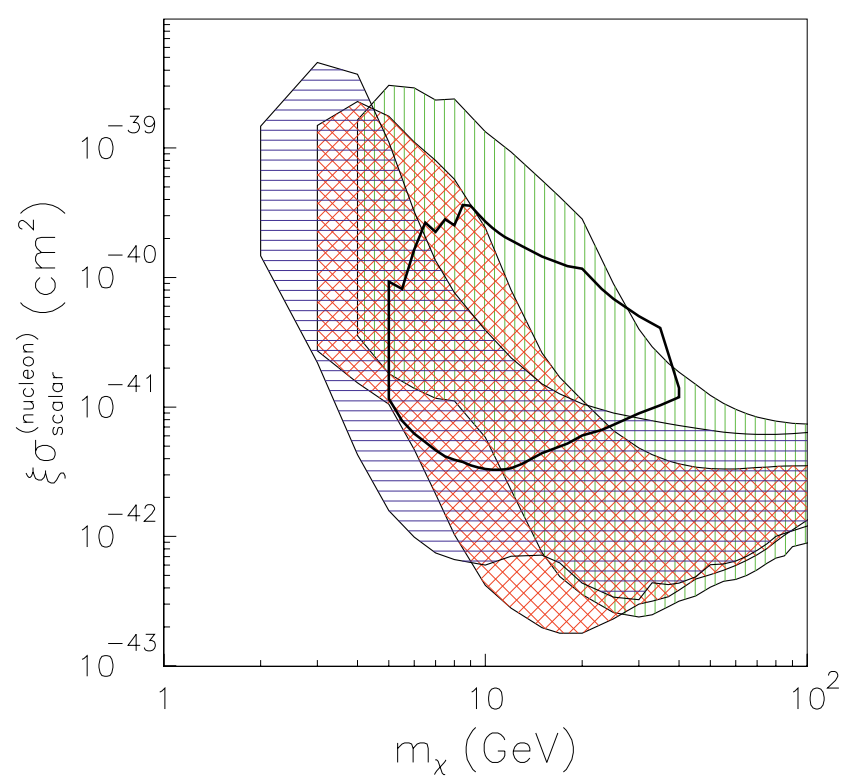

Figure 5. Regions in the nucleon cross section vs DM particle mass plane allowed by DAMA in three different instances for the Na and I quenching factors: i) without including the channeling effect [(green) verticallyhatched region], ii) by including the channeling effect [(blue) horizontally-hatched region)], and iii) without the channeling effect using the energy-dependent $\mathrm{Na}$ and I quenching factors [48] [(red) cross-hatched region]. The velocity distributions and the same uncertainties as in Refs. $[4,5]$ are considered here. The allowed region obtained for the CoGeNT experiment, including the same astrophysical models as in Refs. [4, 5] and assuming for simplicity a fixed value for the Ge quenching factor and a Helm form factor with fixed parameters, is also reported and denoted by a (black) thick solid line. For details see Ref. [48].

DAMA/LIBRA set-up is: i) to increase the experimental sensitivity lowering the software energy threshold of the experiment; 2) to improve the investigation on the nature of the Dark Matter particle and related astrophysical, nuclear and particle physics arguments; 3) to investigate other signal features; 4) to improve the sensitivity in the investigation of rare processes other than Dark Matter as done by the former DAMA/NaI apparatus in the past [12] and by itself so far [19, 20]. This requires long and heavy full time dedicated work for reliable collection and analysis of very large exposures, as DAMA collaboration has always done.

Since January 2011 the DAMA/LIBRA experiment is again in data taking in the new configuration, named DAMA/LIBRA-phase 2.

\section{References}

[1] R. Bernabei et al. Il Nuovo Cim. A 112, 545 (1999).

[2] R. Bernabei et al. Phys. Lett. B 389, 757 (1996); Phys. Lett. B 424, 195 (1998); Phys. Lett. B 450, 448 (1999); Phys. Rev. D 61, 023512 (2000); Phys. Lett. B 480, 23 (2000); Phys. Lett. B 509, 197 (2001); Eur. Phys. J. C 23, 61 (2002); Phys. Rev. D 66, 043503 (2002); Int. J. Mod. 
Phys. A 21, 1445 (2006); Eur. Phys. J. C 47, 263 (2006); Int. J. Mod. Phys. A 22, 3155 (2007); Eur. Phys. J. C 53, 205 (2008); Phys. Rev. D 77, 023506 (2008); Mod. Phys. Lett. A 23, 2125 (2008).

[3] R. Bernabei et al., Eur. Phys. J. C 18, 283 (2000).

[4] R. Bernabei et al. La Rivista del Nuovo Cimento 26, n.1, 1 (2003).

[5] R. Bernabei et al., Int. J. Mod. Phys. D 13, 2127 (2004).

[6] R. Bernabei et al., Int. J. Mod. Phys. A 21, 1445 (2006).

[7] R. Bernabei et al., Eur. Phys. J. C 47, 263 (2006).

[8] R. Bernabei et al., Int. J. Mod. Phys. A 22, 3155 (2007).

[9] R. Bernabei et al., Eur. Phys. J. C 53, 205 (2008).

[10] R. Bernabei et al., Phys. Rev. D 77, 023506 (2008).

[11] R. Bernabei et al., Mod. Phys. Lett. A 23, 2125 (2008).

[12] See in the publication list in: http://people.roma2.infn.it/dama

[13] P. Belli et al., Astropart. Phys. 5, 217 (1996); Nuovo Cim. C 19, 537 (1996); Phys. Lett. B B387, 222 (1996); Phys. Lett. B 389, 783 (err.) (1996); R. Bernabei et al., Phys. Lett. B 436, 379 (1998); P. Belli et al., Phys. Lett. B 465, 315 (1999); Phys. Rev. D 61, 117301(2000); R. Bernabei et al., New J. of Phys. 2, 15.1 (2000); Phys. Lett. B 493, 12 (2000); Nucl. Instr. \& Meth A 482, 728 (2000); Eur. Phys. J. direct C 11, 1 (2001); Phys. Lett. B 527, 182 (2002); Phys. Lett. B 546, 23 (2002); Beyond the Desert 2003 (Berlin: Springer) p. 365 (2003); Eur. Phys. J. A 27, s01 35 (2006).

[14] R. Bernabei et al., Astropart. Phys. 7, 73 (1997); Nuovo Cim. A 110, 189 (1997); P. Belli et al., Astropart. Phys. 10, 115 (1999); Nucl. Phys. B 563, 97 (1999); R. Bernabei et al., Nucl. Phys. A 705, 29 (2002); P. Belli et al., Nucl. Instr. \& Meth A 498, 352 (2003); R. Cerulli et al., Nucl. Instr. \& Meth A 525, 535 (2004); R. Bernabei et al., Nucl. Instr. \& Meth A 555, 270 (2005); Ukr. J. Phys. 51, 1037 (2006); P. Belli et al., Nucl. Phys. A 789, 15 (2007); Phys. Rev. C 76, 064603 (2007); Phys. Lett. B658, 193 (2008); Eur. Phys. J. A 36, 167 (2008); Nucl. Phys. A 826, 256 (2009); Nucl. Instr. \& Meth A 615, 301 (2010); Nucl. Instr. \& Meth A 626-627, 31 (2011); J. Phys. G: Nucl. Part. Phys. 38, 015103 (2011).

[15] P. Belli et al., Nucl. Instr. \& Meth. A 572, 734 (2007); Nucl. Phys. A 806, 388 (2008); Nucl. Phys. A 824, 101 (2009); Proceed. of the Int. Conf. NPAE 2008 (ed. INR-Kiev, Kiev), p. 473 (2009); Eur. Phys. J. A 42, 171 (2009); Nucl. Phys. A 846, 143 (2010); Nucl. Phys. A 859, 126 (2011); Phys. Rev. C 83, 034603 (2011); Eur. Phys. J. A 47, 91 (2011).

[16] R. Bernabei et al., Nucl. Instr. \& Meth. A 592, 297 (2008).

[17] R. Bernabei et al., Eur. Phys. J. C 56, 333 (2008).

[18] R. Bernabei et al., Eur. Phys. J. C 67, 39 (2010).

[19] R. Bernabei et al., Eur. Phys. J. C 62, 327-332 (2009).

[20] R. Bernabei et al., Eur. Phys. J. C 72, 1920 (2012).

[21] R. Bernabei et al., Phys. Lett. B 509, 197 (2001); P. Belli et al., Phys. Rev. D 66, 043503 (2002); D.G. Cerdeno et al., J. Cosm. Astrop. Phys. 06, 008 (2007); P. Belli et al., Phys. Rev. D 84, 055014 (2011); A. Bottino et al., Phys. Rev. D 81, 107302 (2010); N. Fornengo et al., Phys. Rev. D 83, 015001 (2011); A. Liam Fitzpatrick et al., Phys. Rev. D 81, 115005 (2010); D. Hooper et al., Phys. Rev. D 82, 123509 (2010); J.F. Gunion et al., arXiv:1009.2555; A.V. Belikov et al., Phys. Lett. B 705, 82 (2011); A. Bottino et al., Phys. Rev. D 85, 095013 (2012).

[22] C. Arina and N. Fornengo., J. High Energy Phys. 0711, 029 (2007); D.G. Cerdeno et al., Phys. Rev. D 79, 023510 (2009); D.G. Cerdeno et al., J. Cosm. Astrop. Phys. 08, 032 (2009); G. Belanger et al., J. High Energy Phys. 1107, 083 (2011). 
[23] R. Bernabei et al., Eur. Phys. J. C 23, 61 (2002).

[24] S. Chang et al., Phys. Rev. D 79, 043513 (2009); S. Chang et al., Phys. Rev. Lett. 106, 011301 (2011); S. Chang et al., J. Cosm. Astrop. Phys. 08, 018 (2010).

[25] R. Foot, Phys. Rev. D 81, 087302 (2010); R. Foot, Phys. Lett. B 703, 7 (2011); R. Foot, Phys. Rev. D 82, 095001 (2010).

[26] Y. Bai and P.J. Fox, J. High Energy Phys. 0911, 052 (2009).

[27] J. Alwall et al., Phys. Rev. D 81, 114027 (2010).

[28] M.Yu. Khlopov et al., arXiv:1003.1144; M.Yu. Khlopov et al., Int. J. Mod. Phys. D 19, 1385 (2010).

[29] S. Andreas et al., Phys. Rev. D 82, 043522 (2010).

[30] V. Barger et al., Phys. Rev. D 82, 035019 (2010).

[31] P.W. Graham et al., Phys. Rev. D 82, 063512 (2010).

[32] B. Batell et al., Phys. Rev. D 79, 115019 (2009).

[33] E. Del Nobile et al., Phys. Rev. D 84, 027301 (2011).

[34] J.L. Feng et al., Phys. Lett. B 703, 124 (2011); M.T. Frandsen et al., Phys. Rev. D 84, 041301 (2011).

[35] M.S. Boucenna and S. Profumo, Phys. Rev. D 84, 055011 (2011); Y.G. Kim and S. Shin, J. High En. Phys. 05, 036 (2009).

[36] M.R. Buckley et al., Phys. Lett. B 703, 343 (2011); M.R. Buckley et al., Phys. Lett. B 702, 216 (2011).

[37] J.L. Feng et al., Phys. Rev. Lett. 91, 011302 (2003); J.L. Feng et al., Phys. Rev. D 70, 075019 (2004).

[38] D.J.H. Chung et al., Phys. Rev. D 64, 043503 (2001).

[39] Y. Mambrini, J. Cosm. Astrop. Phys. 07, 009 (2011); Y. Mambrini, J. Cosm. Astrop. Phys. 09, 022 (2010); J. Kopp et al., J. Cosm. Astrop. Phys. 02, 014 (2010); S. Shin, arXiv:1011.6377; C. Arina et al., J. Cosm. Astrop. Phys. 09, 022 (2011); W.Y. Keung et al., Phys. Rev. D 82, 115019 (2010).

[40] D. Smith and N. Weiner, Phys. Rev. D 64, 043502 (2001); D. Tucker-Smith and N. Weiner, Phys. Rev. D 72, 063509 (2005).

[41] A.K. Drukier, K. Freese, and D.N. Spergel, Phys. Rev. D 33, 3495 (1986); K. Freese, J. A. Frieman and A. Gould, Phys. Rev. D 37, 3388 (1988).

[42] K. Freese et al., Phys. Rev. D 71, 043516 (2005); Phys. Rev. Lett. 92, 111301 (2004).

[43] F. S. Ling, P. Sikivie and S. Wick, Phys. Rev. D 70, 123503-19 (2004).

[44] R. Bernabei et al., AIP Conf. Proceed. 1223, 50 (2010) [arXiv:0912.0660].

[45] R. Bernabei et al., J. Phys.: Conf. Ser. 203, 012040 (2010) [arXiv:0912.4200]; http://taup2009.lngs.infn.it/slides/jul3/nozzoli.pdf, talk given by F. Nozzoli.

[46] R. Bernabei et al., arXiv:1007.0595 to appear on Proceed. of the Int. Conf. Frontier Objects in Astrophysics and Particle Physics, May 2010, Vulcano, Italy.

[47] R. Bernabei et al., Eur. Phys. J. C 72, 2064 (2012).

[48] P. Belli et al., Phys. Rev. D 84, 055014 (2011).

[49] A.Bottino et al., arXiv:1112.5666; A. Bottino et al., Phys. Rev. D 81, 107302 (2010); N. Fornengo et al., Phys. Rev. D 83, 015001 (2011); A.L. Fitzpatrick et al., arXiv:1003.0014; D. Hooper et al., arXiv:1007.1005v2; D. G. Cerdeno and O. Seto, JCAP 0908, 032 (2009); D. G. Cerdeno, C. Munoz and O. Seto, Phys. Rev. D 79, 023510 (2009); D. G. Cerdeno et al., JCAP 0706, 008 (2007); J.F. Gunion et al., arXiv:1009.2555; A.V. Belikov et al., arXiv:1009.0549; 
C. Arina and N. Fornengo, JHEP 11, 029 (2007); G. Belanger et al, arXiv:1105.4878; S. Chang et al., Phys. Rev. D 79, 043513 (2009); S. Chang et al., arXiv:1007.2688; R. Foot, arXiv:1001.0096, arXiv:1106.2688, Phys. Rev. D 82, 095001 (2010); Y. Mambrini, JCAP, 1107, 009 (2011); JCAP 1009, 022 (2010); Y. Bai and P.J. Fox, JHEP 0911, 052 (2009); J. Alwall et al., arXiv:1002.3366; M. Yu Khlopov et al., arXiv:1003.1144; S. Andreas et al., Phys. Rev. D 82, 043522 (2010); M.S. Boucenna, S. Profumo, arXiv:1106.3368; P.W. Graham et al., Phys. Rev. D 82, 063512 (2010); B. Batell, M. Pospelov and A. Ritz, Phys. Rev. D 79, 115019 (2009); E. Del Nobile et al., Phys. Rev. D 84, 027301 (2011); J. Kopp et al., JCAP 1002, 014 (2010); V. Barger et al., Phys. Rev. D 82, 035019 (2010); S. Chang et al., JCAP 1008, 018 (2010); J.L. Feng et al., Phys. Lett. B 703, 124 (2011); M.T. Frandsen et al., Phys. Rev. D 84, 041301 (2011); Y.G. Kim and S. Shin, JHEP 0905, 036 (2009); S. Shin, arXiv:1011.6377; M.R. Buckley, arXiv:1106.3583; N. Fornengo et al., arXiv:1108.4661; P. Gondolo et al., arXiv:1106.0885; E. Kuflik et al., arXiv:1003.0682; C. Arina et al., arXiv:1105.5121; M.R. Buckley et al., arXiv:1011.1499.

[50] A. Bottino et al., Phys. Rev. D 85, 095013 (2012).

[51] C.E. Aalseth et al., arXiv:1002.4703; arXiv:1106.0650

[52] G. Angloher et al., arXiv:1109.0702

[53] Z. Ahmed et al., Science 327, 1619 (2010).

[54] E. Armengaud et al., Phys. Lett. B 702, 329 (2011).

[55] E. Aprile et al., Phys. Rev. Lett. 105, 131302 (2010).

[56] R. Bernabei et al., Liquid Noble gases for Dark Matter searches: a synoptic survey, Exorma Ed., Roma, ISBN 978-88-95688-12-1, pp. 1-53 (arXiv:0806.0011v2) (2009).

[57] J.I. Collar and D.N. McKinsey, arXiv:1005.0838; arXiv:1005.3723; J.I. Collar, arXiv:1006.2031; arXiv:1010.5187; arXiv:1103.3481; arXiv:1106.0653; arXiv:1106.3559

[58] R. Bernabei et al., J. of Inst. 7, P03009 (2012). 